\title{
Phlebotomine Sand Fly Fauna and Leishmania Infection in the Vicinity of the Serra do Cipó National Park, a Natural Brazilian Heritage Site
}

\author{
Rosana Silva Lana, ${ }^{1}$ Érika Monteiro Michalsky, ${ }^{1}$ Consuelo Latorre Fortes-Dias, ${ }^{2}$ \\ João Carlos França-Silva, ${ }^{3}$ Fabiana de Oliveira Lara-Silva, ${ }^{1}$ \\ Ana Cristina Vianna Mariano da Rocha Lima, ${ }^{1}$ Daniel Moreira de Avelar, ${ }^{1}$ \\ Juliana Cristina Dias Martins, ${ }^{4}$ and Edelberto Santos Dias ${ }^{1}$ \\ ${ }^{1}$ Laboratório de Leishmanioses, Centro de Pesquisas René Rachou, Fundação Oswaldo Cruz, \\ Avenida Augusto de Lima 1715, 30190-002 Belo Horizonte, MG, Brazil \\ ${ }^{2}$ Diretoria de Pesquisa e Desenvolvimento, Fundação Ezequiel Dias, 30510-010 Belo Horizonte, MG, Brazil \\ ${ }^{3}$ Departamento de Parasitologia, ICB, Universidade Federal Minas Gerais, 31270-901 Belo Horizonte, MG, Brazil \\ ${ }^{4}$ Secretaria Municipal de Saúde, 35830-000 Jaboticatubas, MG, Brazil \\ Correspondence should be addressed to Edelberto Santos Dias; edel@cpqrr.fiocruz.br
}

Received 10 September 2014; Revised 15 November 2014; Accepted 17 November 2014

Academic Editor: Mehdi Chenik

Copyright ( 2015 Rosana Silva Lana et al. This is an open access article distributed under the Creative Commons Attribution License, which permits unrestricted use, distribution, and reproduction in any medium, provided the original work is properly cited.

\begin{abstract}
In the New World, the leishmaniases are primarily transmitted to humans through the bites of Leishmania-infected Lutzomyia (Diptera: Psychodidae) phlebotomine sand flies. Any or both of two basic clinical forms of these diseases are endemic to several cities in Brazil-the American cutaneous leishmaniasis (ACL) and the American visceral leishmaniasis (AVL). The present study was conducted in the urban area of a small-sized Brazilian municipality (Jaboticatubas), in which three cases of AVL and nine of ACL have been reported in the last five years. Jaboticatubas is an important tourism hub, as it includes a major part of the Serra do Cipó National Park. Currently, no local data is available on the entomological fauna or circulating Leishmania. During the one-year period of this study, we captured 3,104 phlebotomine sand flies belonging to sixteen Lutzomyia species. In addition to identifying incriminated or suspected vectors of ACL with DNA of the etiological agent of AVL and vice versa, we also detected Leishmania DNA in unexpected Lutzomyia species. The expressive presence of vectors and natural Leishmania infection indicates favorable conditions for the spreading of leishmaniases in the vicinity of the Serra do Cipó National Park.
\end{abstract}

\section{Introduction}

Leishmaniases are a complex of parasitic diseases caused by flagellated protozoa belonging to genus Leishmania Ross, 1903. About 310 million individuals are at risk of contracting any of the various clinical forms of leishmaniasis, and some 2 million new cases occur yearly [1]. In the New World, the leishmaniases are primarily transmitted to humans through the bites of Lutzomyia (Diptera: Psychodidae) phlebotomine sand flies [2]. Two basic clinical forms of leishmaniases are known-the American cutaneous leishmaniasis (ACL) and the American visceral leishmaniasis (AVL). Each form has different Leishmania species as etiological agents and distinct Lutzomyia species as transmitting vectors. Only the phlebotomine females play a role in the infection process due to their bloodsucking feeding habits.

Leishmania (Viannia) braziliensis, Le. (V.) guyanensis, and Le. (Leishmania) amazonensis are the most important causative agents of ACL [3]. A number of phlebotomine sand fly species have been incriminated as ACL vectors, including Lutzomyia (Nyssomyia) intermedia (Lutz \& Neiva, 1912), Lu. migonei (França, 1920), Lu. (N.) whitmani 
(Antunes \& Coutinho, 1939), Lu. (Pintomyia) fisheri (Pinto, 1926), Lu. (P.) pessoai (Coutinho \& Barreto, 1940), and Lu. (N.) flaviscutellata (Mangabeira, 1942) [4].

Leishmania (Leishmania) infantum (syn. Le. chagasi) is recognized as etiological agent of AVL [5]. In Brazil, two Lutzomyia species are involved in AVL transmissionLutzomyia longipalpis (Lutz \& Neivai, 1912) as the primary and $\mathrm{Lu}$. cruzi (Mangabeira, 1938) as the secondary vector [6].

Between 2009 and 2013, about 113,600 and 18,000 new cases of ACL and AVL, respectively, were compulsorily reported to the Brazilian Ministry of Health [7]. In largeand medium-sized cities, public health services have been put in place, providing the necessary logistic support to epidemiological studies and control actions. In smaller towns, however, this support is incipient.

The present study was developed in Jaboticatubas, a small-sized municipality and an important tourism hub located in the Brazilian state of Minas Gerais. The municipality includes about $65 \%$ of the area of the Serra do Cipó National Park, an important natural Brazilian heritage site. This park is known for its rich and diversified flora and fauna, tracking trails, waterfalls, and archaeological sites with cave paintings. As it attracts hundreds of visitors every year, it is an important economic asset. In the 2009-2013 period, three human cases of AVL and nine cases of ACL were reported in Jaboticatubas [7]. Presently, the transmission potential in the region is unknown. Therefore, the aim of this work was to survey the local presence of possible phlebotomine sand fly vectors and of etiological agents of leishmaniases. To our knowledge, this is the first entomological study developed in Jaboticatubas and in Serra do Cipó National Park surroundings.

\section{Material and Methods}

2.1. Study Area. Jaboticatubas $\left(19^{\circ} 30^{\prime} 49^{\prime \prime} \mathrm{S}, 43^{\circ} 44^{\prime} 42^{\prime \prime} \mathrm{W}\right)$ is located at the Serra do Espinhaço, in Southeastern Brazil. The municipality extends across a $1,114.1 \mathrm{~km}^{2}$ area, at an average altitude of $772 \mathrm{~m}$. According to the latest survey, it had a population of 17,134 inhabitants, which is equivalent to 15.4 inhabitants $/ \mathrm{km}^{2}$ [8]. The climate is tropical, characterized by cool summers and a well-defined dry season. This mountainous region is rich in quartzite rocks and outcrops of limestone, with predominance of sandy soil with rock fields, covered by riparian forest and savannah vegetation [9].

2.2. Entomological Survey. The entomological captures that yielded data for the subsequent analyses were performed from May 2012 to April 2013, using HP light traps [10] placed in the peridomicile of ten houses in the urban area of Jaboticatubas. The trapping sites were labeled from A to J (Figure 1). The houses included in the study were selected based on the environmental conditions that favor the rearing of phlebotomine sand flies - such as shadowed areas, presence of domestic animals, and fruit trees-and previous reports of canine cases of leishmaniases in the neighborhood. The residents of the selected houses were informed of the project objectives and voluntarily signed a statement of informed consent prior to commencing the entomological captures. The trapping sites were georeferenced using a GARMIM-ETREX GPS.

The captures took place from 6:00 pm to 8:00 am, on three consecutive nights, always in the first week of each month. All captured male phlebotomine specimens were preserved in $70 \%$ ethanol and taken to the laboratory for species identification, while the females were placed in microtubes containing $6 \% \mathrm{DMSO}$ and stored at $-20^{\circ} \mathrm{C}$ until being required. For species identification, the head and the last three abdominal segments of every female were removed and slide-mounted with Berlese liquid. The remaining body parts were pooled and used for DNA extraction. The males were also slide-mounted with Berlese liquid.

We identified the phlebotomine sand flies of both genders using specific descriptions and taxonomic keys, as described by Young and Duncan [11]. Specimens that could not be identified due to missing or incomplete characters were considered Lutzomyia spp. Lu. sallesi and Lu. cortelezzii were considered cortelezzii complex, owing to the morphological similarity of their females [12]. Due to their high morphological resemblance, taxonomic identification of Brumptomyia females stopped at the genus level.

2.3. Climate Data. The monthly average maximum temperature $\left({ }^{\circ} \mathrm{C}\right)$, total rainfall $(\mathrm{mm})$, and relative humidity $(\%)$ data were sourced from the nearest meteorological station (fifth district of the Brazilian Institute of Meteorology, Belo Horizonte, MG). We employed the Spearman correlation analysis to evaluate the influence of climate variables on the population density of phlebotomine sand flies, using the Prism 6 software (GraphPad Inc., USA) with a 5\% significance level. The results were expressed as the simple Spearman correlation coefficient (rs) for each pair of variables.

2.4. DNA Extraction from Phlebotomine Sand Flies. We extracted the total DNA from phlebotomine sand fly females using the Cell and Tissue Genomic Prep kit (GE Healthcare), after combining in a single pooled sample up to ten specimens of the same species, captured in the same month at the same capture site. Each DNA sample was identified by a number followed by the Lutzomyia species it was extracted from. The reliability of the DNA extraction was ensured by the amplification with genus-specific primers for Lutzomyia (5Llcac 5' GTG GCC GAA CAT AAT GTT AG $3^{\prime}$ and 3Llcac $5^{\prime}$ CCA CGA ACA AGT TCA ACA TC $3^{\prime}$ ) in the cacophony IVS6 region, as described by other authors [13].

2.5. Nested PCR for Leishmania (LnPCR). The test for the presence of Leishmania DNA in the phlebotomine sand flies was carried out by Leishmania nested PCR ( $\operatorname{n}$ PCR), specifically targeting the SSUrRNA gene [14-16]. Briefly, total DNA extracted from phlebotomine sand flies was first amplified with specific primers for the order Kinetoplastida but not exclusively for Leishmania. The resulting product of 603 bp was used as a template in the second PCR amplification in the presence of Leishmania-specific primers. Positive samples for Leishmania DNA showed a $353 \mathrm{bp}$ fragment that was visualized under UV light after electrophoresis on 


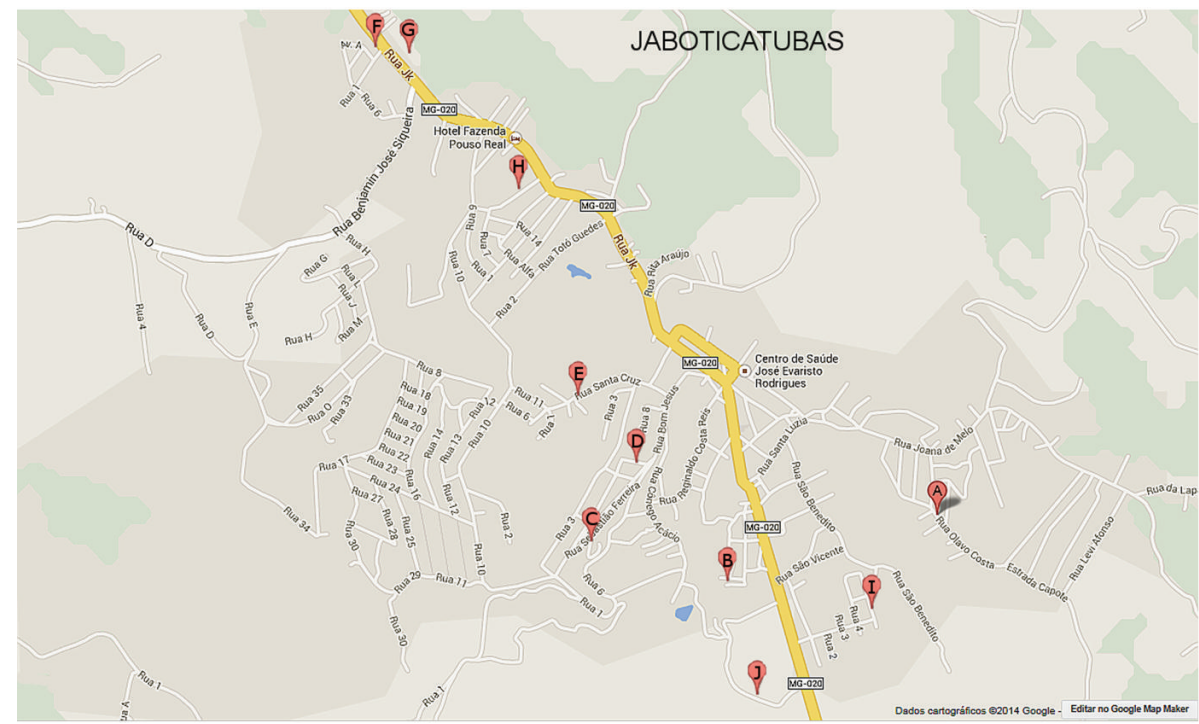

FIGURE 1: Spatial distribution of the entomological trapping sites in the urban area of Jaboticatubas (Minas Gerais state, Brazil). The study was conducted between May 2012 and April 2013.

$2 \%$ agarose gel and ethidium bromide staining. Negative (no DNA) and positive-DNA extracted from Le. infantum (MHOM/BR74/PP75)—controls were run in parallel.

2.6. Leishmania Species Identification and Phylogenetic Tree. The fragments amplified by $L n P C R$ were purified from agarose gels using a commercial kit (QIAquick Gel Extraction Kit, QIAGEN) and submitted to DNA sequencing, in both directions, using an appropriate kit (BigDye Terminator v3.1 Cycle) and the Megabace analyzer (GE HealthCare). Sequence editions and alignments against Leishmania DNA sequences [Le. braziliensis (M80292.1), Le. amazonensis (M80293.1), and Le. chagasi (M81430.1)] deposited in the GenBank database were performed using BioEdit tools (http://www.mbio.ncsu.edu/bioedit/bioedit.html) and the MacVector v. 11.0.2 software (MacVector Inc., Informax Inc., USA).

A consensus phylogenetic tree was constructed for Leishmania in the infected Lutzomyia phlebotomine sand flies using the distance-based unweighted pair group method with arithmetic mean (UPMGA). The analysis was performed with default parameters [bootstrap of 1,000 replicates, systematic tie breaking, Kimura 2-parameter, gamma correction off, estimate transversion ratio $(\mathrm{Av} .=0.000)$, and proportionally distributed gaps] of the MacVector v. 11.0.2 software (MacVector Inc., Informax Inc., USA).

2.7. Minimum Infection Rates by Leishmania in the Phlebotomine Sand Flies. The minimum infection rates (MIR) by Leishmania in the captured phlebotomine sand flies were calculated as the ratio of the number of positive pools of each sand fly species and the number of specimens in that pool, multiplied by 100 [17].

\section{Results}

3.1. Phlebotomine Sand Fly Survey. During the 12-month period included in the study, we captured 3,104 phlebotomine sand flies belonging to two genera, Brumptomyia and Lutzomyia (Table 1), with the overall male/female ratio of 2.3. Among the sixteen different species belonging to Lutzomyia genus, six are incriminated vectors of leishmaniases, namely, Lu. fischeri, Lu. intermedia, Lu. migonei, Lu. pessoai, and Lu. whitmani (as vectors of ACL) and Lu. longipalpis (vector of AVL) (Table 1). Three of the ten entomological trapping sites-D, G, and J-comprised about $86 \%$ of the total number of phlebotomine sand flies captured (Table 2). In addition, at these sites, at least one incriminated vector of leishmaniases as dominant species was observed (Figure 2).

3.2. Seasonal Variation Is the Phlebotomine Sand Fly Population. A positive correlation was found between the phlebotomine population density and the climate variables with correlation coefficients (rs) of 0.6719 for rainfall, 0.8792 for temperature, and 0.2035 for humidity. The correlation was statistically significant only for rainfall and temperature with $P$ values of 0.0194 and 0.0003 , respectively.

It is important to note the expressive increase in the population density during the short rainy season (November 2012-January 2013), accounting for 59\% of the total number of specimens captured. As can be seen in Figure 3, a marked population increase occurred between two rainfall peaks.

3.3. Detection of Leishmania DNA in Phlebotomine Sand Flies. The DNA tests were performed on 249 species-specific pooled samples of Lutzomyia females, aiming to identify presence of the Lutzomyia cacophony gene. The expected 220 bp fragment was present in all samples (data not shown), 
TABLE 1: Phlebotomine sand flies captured in the municipality of Jaboticatubas, in the Brazilian state of Minas Gerais, during the May 2012 April 2013 study period, using HP-like light traps. The proven or incriminated vectors of leishmaniases are marked with *

\begin{tabular}{|c|c|c|c|c|c|}
\hline \multirow{2}{*}{ Species } & & \multicolumn{3}{|c|}{ Number of specimens } & \multirow{2}{*}{ Relative percentage } \\
\hline & & Males & Females & Both genders & \\
\hline Brumptomyia sp. & & 0 & 2 & 2 & 0.1 \\
\hline cortelezzii complex & & 76 & 105 & 181 & 5.8 \\
\hline Lutzomyia aragaoi & & 1 & 1 & 2 & 0.1 \\
\hline Lu.evandroi & & 0 & 1 & 1 & 0.0 \\
\hline Lu. fischeri ${ }^{*}$ & & 1 & 1 & 2 & 0.1 \\
\hline Lu. intermedia* & & 376 & 77 & 453 & 14.6 \\
\hline Lu. lenti & & 482 & 260 & 742 & 23.9 \\
\hline Lu. lloydi & & 0 & 1 & 1 & 0.0 \\
\hline Lu. longipalpis* & & 197 & 50 & 247 & 8.0 \\
\hline Lu. lutziana & & 2 & 4 & 6 & 0.2 \\
\hline Lu. migonei ${ }^{*}$ & & 31 & 5 & 36 & 1.2 \\
\hline Lu. pessoai* & & 194 & 76 & 270 & 8.7 \\
\hline Lu. quinquefer & & 1 & 0 & 1 & 0.0 \\
\hline Lu. renei & & 0 & 2 & 2 & 0.1 \\
\hline Lu. sordellii & & 4 & 28 & 32 & 1.0 \\
\hline Lu. termitophila & & 1 & 7 & 8 & 0.3 \\
\hline Lu. whitmani ${ }^{*}$ & & 785 & 264 & 1,049 & 33.8 \\
\hline Lutzomyia spp. & & 12 & 57 & 69 & 2.2 \\
\hline \multirow{2}{*}{ Total } & Number & 2,163 & 941 & 3,104 & - \\
\hline & $\%$ & 69.7 & 30.3 & - & 100.0 \\
\hline
\end{tabular}

TABLE 2: Number of phlebotomine sand flies captured monthly, using HP light traps, in Jaboticatubas, in the Brazilian state of Minas Gerais, during the May 2012-April 2013 study period.

\begin{tabular}{|c|c|c|c|c|c|c|c|}
\hline \multirow{2}{*}{ Year } & \multirow{2}{*}{ Month } & \multicolumn{6}{|c|}{ Number of specimens per trapping site } \\
\hline & & $\mathrm{D}$ & G & $\mathrm{J}$ & Others & Total & $\%$ \\
\hline \multirow{8}{*}{2012} & May & 25 & 13 & 31 & 6 & 75 & 2.4 \\
\hline & June & 5 & 51 & 21 & 15 & 92 & 3.0 \\
\hline & July & 12 & 55 & 44 & 13 & 124 & 4.0 \\
\hline & August & 21 & 18 & 16 & 16 & 71 & 2.3 \\
\hline & September & 20 & 87 & 5 & 12 & 124 & 4.0 \\
\hline & October & 11 & 148 & 22 & 40 & 221 & 7.1 \\
\hline & November & 70 & 355 & 30 & 88 & 543 & 17.5 \\
\hline & December & 238 & 256 & 529 & 95 & 1,118 & 36.0 \\
\hline \multirow{4}{*}{2013} & January & 7 & 61 & 51 & 41 & 160 & 5.2 \\
\hline & February & 6 & 0 & 196 & 29 & 231 & 7.4 \\
\hline & March & 3 & 115 & 69 & 46 & 233 & 7.5 \\
\hline & April & 11 & 21 & 39 & 41 & 112 & 3.6 \\
\hline Total & & 429 & 1,180 & 1,053 & 442 & 3,104 & 100.0 \\
\hline
\end{tabular}

confirming the reliability of the DNA extraction from the phlebotomine sand flies.

The 353 bp fragment characteristic of Leishmania genus was detected in 32 of the aforementioned 249 pooled samples of Lutzomyia (Table 3), with an overall MIR of 3.4\%. Infected Lutzomyia specimens were captured from every trapping site (data not shown).
3.4. Specific Determination of the Infecting Leishmania in Lutzomyia Sand Flies. The nucleotide (nt) sequences of the 353 bp Leishmania fragments in the infected Lutzomyia phlebotomine sand flies were compatible with either Le. infantum or Le. braziliensis. Le. amazonensis was not detected in our samples. The alignment region containing the discriminating nucleotide mutations for the two Leishmania 
TABLE 3: Lutzomyia species carrying Leishmania DNA captured in Jaboticatubas (MG, Brazil). Suspected or incriminated vectors of leishmaniases are marked with *. Infecting Leishmania species were determined by nested PCR (LnPCR). Le.infantum and Le.braziliensis are etiological agents of the American visceral (AVL) and of the American cutaneous (ACL) leishmaniases, respectively. Study period: May 2012-April 2013.

\begin{tabular}{|c|c|c|c|c|}
\hline \multirow{2}{*}{ Lutzomyia species } & \multicolumn{4}{|c|}{ Leishmania DNA identification } \\
\hline & Le. infantum & Le. braziliensis & Leishmania sp. & Total \\
\hline cortelezzii complex & 1 & 8 & 2 & 11 \\
\hline Lu. intermedia* & 0 & 2 & 0 & 2 \\
\hline Lu. lenti & 1 & 2 & 1 & 4 \\
\hline Lu. longipalpis* & 1 & 4 & 1 & 6 \\
\hline Lu. migonei ${ }^{*}$ & 0 & 0 & 1 & 1 \\
\hline Lu. pessoai ${ }^{*}$ & 0 & 3 & 1 & 4 \\
\hline Lu. sordellii & 0 & 1 & 0 & 1 \\
\hline Lu. whitmani* & 1 & 1 & 1 & 3 \\
\hline Total & 4 & 21 & 7 & 32 \\
\hline
\end{tabular}

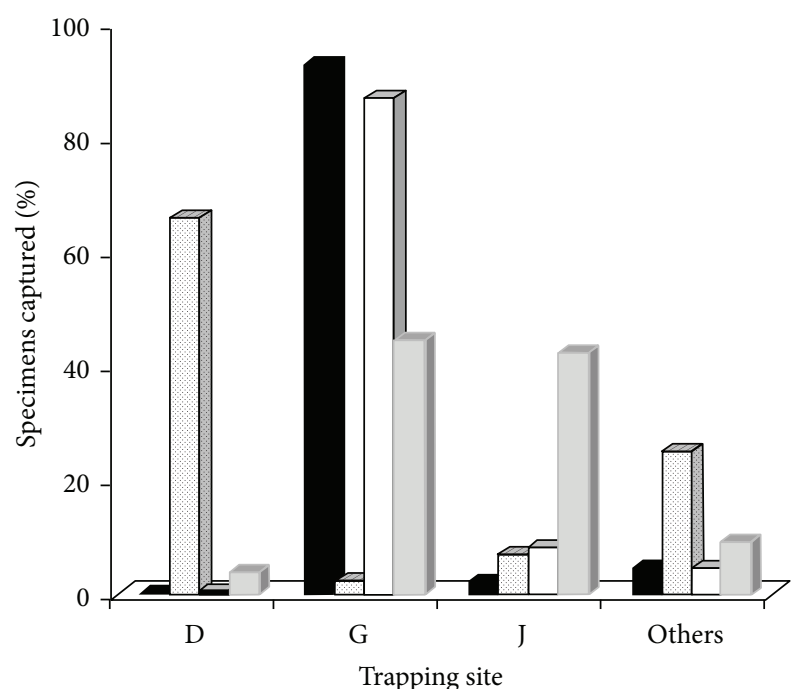

Lu. intermedia

Lu. pessoai

Lu. longipalpis

Lu. whitmani

FIGURE 2: Distribution of vector species of leishmaniases according to entomological trapping sites in Jaboticatubas (Minas Gerais state, Brazil), between May 2012 and April 2013.

species is shown in the Supplementary Table available online at http://dx.doi.org/10.1155/2015/385493. A phylogenetic tree generated two clusters corresponding to Le. infantum- and Le. braziliensis-infected Lutzomyia samples (Figure 4). The infecting Leishmania species was successfully determined in 28 of the 32 samples of Lutzomyia (Table 3).

Le. braziliensis, an etiological agent of ACL, was detected in eight Lutzomyia species, namely, Lu. intermedia, Lu. pessoai, Lu. whitmani, Lu. longipalpis, cortelezzii complex, Lu. lenti, and Lu. sordellii. However, only the first three species are incriminated vectors of ACL, whereas $L u$. longipalpis is vector of AVL. On the other hand, the causative agent of AVL-Le. infantum - was present not only in Lu. longipalpis, the proven vector of AVL, but also in the cortelezzii complex, Lu. lenti, Lu. longipalpis, Lu. migonei, Lu. pessoai, and Lu. whitmani.

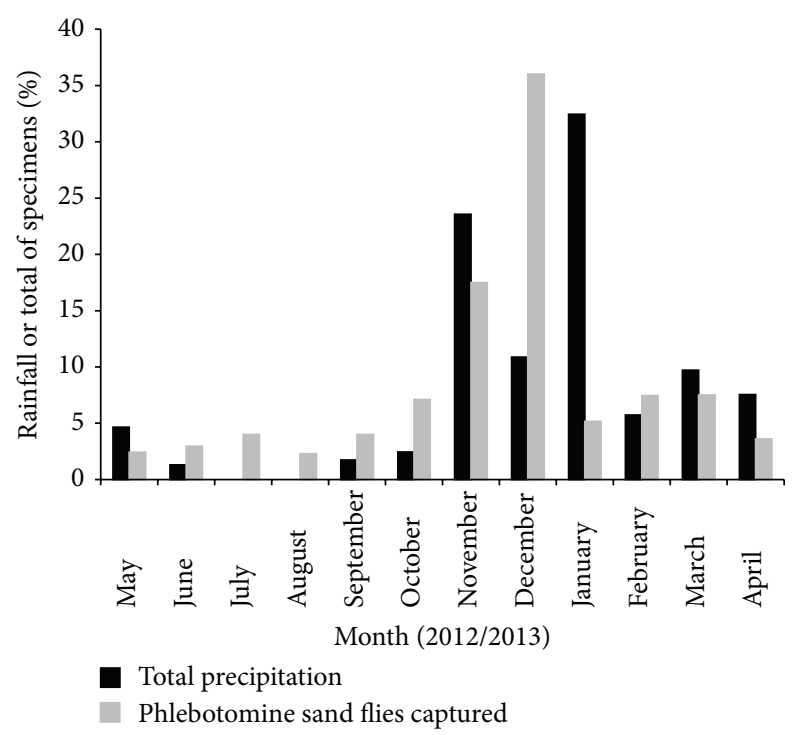

FIGURE 3: Influence of rainfall (black bars) on the population density of phlebotomine sand flies (grey bars) in the municipality of Jaboticatubas, Minas Gerais state, Brazil. The study was conducted between May 2012 and April 2013.

\section{Discussion}

During the one-year study in Jaboticatubas, we captured an expressive number of Lutzomyia phlebotomine sand flies, belonging to sixteen different species. In accordance with the findings of several studies previously conducted in other Brazilian areas, the overall population density of Lutzomyia tended to increase significantly with rainfall and temperature, with most of the specimens captured during the short rainy season [18-28]. This profile may favor the planning of insect control actions.

Six incriminated vectors of leishmaniases were present, namely, Lu. fischeri, Lu. intermedia, Lu. migonei, Lu. pessoai, and $L u$. whitmani (known vectors of ACL) and Lu. longipalpis (vector of AVL). Lu. whitmani was the most abundant species, accounting for $1 / 3$ of the total number of the specimens 


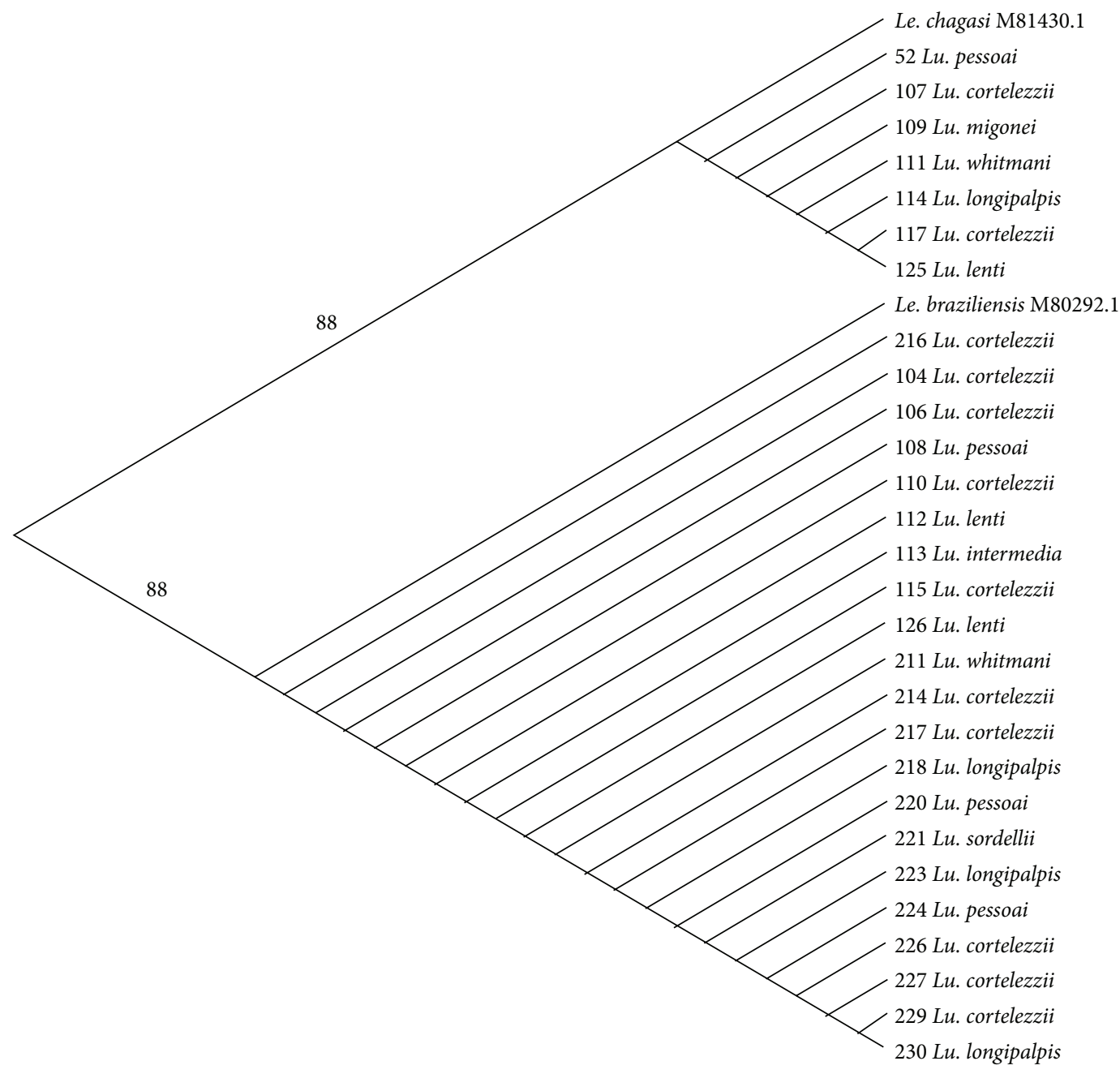

FIgURE 4: Phylogenetic tree (UPGMA) of Leishmania DNA identified in Lutzomyia phlebotomine sand flies captured in Jaboticatubas, state of Minas Gerais, Brazil. The bootstrap values are indicated on the branches. References: Le. chagasi M81430.1 and Le. braziliensis M80292.1. Test samples are identified by numbers followed by the Lutzomyia species carrying the Leishmania DNA. Study period: May 2012 to April 2013.

captured in our study. This is a widely distributed species in both rural and urban areas and is amongst the most important vectors of ACL in Brazil [4, 29-35]. We found evidence that $\mathrm{Lu}$. whitmani is separately infected by etiological vectors of both ACL and AVL, that is, Le. braziliensis and Le. infantum, respectively, in line with previous reports $[4,36,37]$.

The second most numerous species in Jaboticatubas was Lu. lenti. This species has been commonly observed in savannah areas, where domestic animals, poultry in particular, are present in large numbers [38]. Although Lu. lenti appeared refractory to experimental Leishmania infection [39], natural infection lacking specific Leishmania identification has been reported by other authors [40]. We found $L u$. lenti separately infected by Le. infantum and by Le. braziliensis.

Lu. intermedia is an important vector of ACL in the Southeast of Brazil [41-44] and, in this study, it was captured at a representative proportion (14.8\%). The species is well adapted to various habitats, from forested to fully human-modified environments, particularly in areas where deforestation is gradually changing the epidemiological profile [4], as is the case in Jaboticatubas.

$\mathrm{Lu}$. pessoai, another frequent species in our captures (8.1\%), was suspected to be involved in ACL transmission in the $40 \mathrm{~s}[45,46]$. Although this hypothesis was not subsequently confirmed, we found Le. braziliensis DNA in that species.

Specimens belonging to the cortelezzii complex, which are not amongst the incriminated leishmaniases vectors, were found to be separately infected by Le. braziliensis and Le. infantum. This is in accordance with previous reports in Brazil and Argentina [37, 47-49].

In addition to the most frequent Lutzomyia species discussed above, we also captured Lu. evandroi, Lu. quinquefer, Lu. migonei, Lu. fischeri, and Lu. sordellii-albeit in smaller numbers. Lu. migonei has been associated with ACL incidence in the Brazilian state of São Paulo [42] and some authors suggested that it could act as a secondary vector 
in the transmission of AVL, particularly in areas where $L u$. longipalpis is absent [50-53]. Unfortunately, we were unable to determine the infecting Leishmania DNA, at specific level, in Lu. migonei.

Lu. fischeri is suspected as a secondary vector of ACL due to its high level of anthropophily and abundance in deforested areas, where sporadic cases of ACL have been reported [50]. However, there are no present or previous reports of natural Leishmania infection in that species.

In a previous study on spatial distribution of sand flies in the Brazilian state of Pernambuco, $\mathrm{Lu}$. sordellii was found almost exclusively in forested areas [54]. In the past, $L u$. sordellii was included in a group characterized by preferential feeding on cold-blooded animals, comprising Lu. quinquefer, among others [38]. Nevertheless, Lu. quinquefer was previously reported to be naturally infected by Leishmania sp. in Argentina [55] and, in the present study, Lu. sordellii infected by Le. braziliensis was identified.

Lutzomyia carrying Leishmania DNA were captured at every trapping site, indicating the wide distribution of the vector species throughout the urban area of Jaboticatubas. In three trapping sites (D, G, and J), the greatest population densities of Lutzomyia were recorded, with dominance of at least one leishmaniases vector. Lu. intermedia and $\mathrm{Lu}$. whitmani were dominant in less-modified sites situated at the periphery of the urban area ( $\mathrm{G}$ and $\mathrm{J}$ ), whereas Lu. longipalpis was dominant at the center of Jaboticatubas, which is more urbanized (trapping site D). Although Lu. longipalpis was captured at much lower percentages than previously reported for other cities $(72-92 \%)$ [22, 27, 28], the species dominance presently observed is compatible with the known adaptation of $\mathrm{Lu}$. longipalpis to peridomiciles in human-modified areas. In addition to Le. infantum (AVL agent), we also captured Lu. longipalpis that was separately infected by Le. braziliensis (ACL agent).

Phylogenetic analysis revealed the clustering of the two circulating Leishmania, independently of the carrying Lutzomyia species. The finding of incriminated or suspected vectors of ACL with DNA of the etiological agent of AVL, and vice versa, suggests permissivity of some phlebotomine sand fly species to the infection by different Leishmania species. Furthermore, Leishmania DNA was identified in unexpected Lutzomyia species. Clearly, further studies are required to elucidate the vectorial competence of unsuspected phlebotomine sand flies species as well as the cross-infection of ACL/AVL vectors and parasites. Leishmania DNA findings do not imply that the infected Lutzomyia might play a role in leishmaniases transmission. According to Killick-Kendrick [56], four criteria must be fulfilled before incriminating any given species as vector of a zoonotic disease: (1) feeding on humans and on the animal reservoir, (2) providing support for the parasites after ingestion and expulsion of the infected blood meal, (3) displaying parasites indistinguishable from those isolated from patients, and (4) having the ability to transmit the parasite by biting.

The overall MIR in Jaboticatubas is comparable to those reported for endemic areas of leishmaniases [57, 58]. Moreover, the abundance and diversity of Lutzomyia species associated with the presence of infection by Le. braziliensis or
Le. infantum indicate favorable conditions for the spreading of both ACL and AVL in an important tourism hub of Brazil. The presence of Leishmania DNA in unexpected Lutzomyia species and the cross-infection of etiological agent and vectors of AVL and ACL are a public health concern that deserves immediate attention. Further studies are needed on phlebotomine sand flies and Leishmania infection for a better understanding of the transmission cycle of leishmaniases in Jaboticatubas. Nevertheless, based on the population density of Lutzomyia and on the proved presence of etiological agents of leishmaniases, this tourism hub deserves special consideration, as timely and effective action may prevent spreading of these diseases to an even greater number of individuals.

\section{Conflict of Interests}

The authors declare that no conflict of interests exists regarding the publication of this paper.

\section{Acknowledgments}

The authors acknowledge the financial support of the Brazilian agencies Fundação de Amparo à Pesquisa de Minas Gerais (FAPEMIG) and Conselho Nacional de Pesquisa e Desenvolvimento Tecnológico (CNPq).

\section{References}

[1] World Health Organization (WHO), Leishmaniasis: background information, Geneva, Switzerland, World Health Organization (WHO), http://apps.who.int/iris/bitstream/10665/ 44412/1/WHO_TRS_949_eng.pdf?ua=1.

[2] R. Killick-Kendrick, M. Maroli, and M. Killick-Kendrick, "Bibliography on the colonization of phlebotomine sandflies," Parassitologia, vol. 33, pp. 321-333, 1991.

[3] E. C. S. Vale and T. Furtado, "Cutaneous leishmaniasis in Brazil: historic revision, expansion and ethiology," Anais Brasileiros de Dermatologia, vol. 80, pp. 421-428, 2005.

[4] R. Lainson and E. F. Rangel, "Ecology of leishmaniasis," in Flebotomíneos do Brasil, E. F. Rangel and R. Lainson, Eds., pp. 291-309, Editora Fiocruz, Rio de Janeiro, Brazil, 2003.

[5] K. Kuhls, M. Z. Alam, E. Cupolillo et al., "Comparative microsatellite typing of new world Leishmania infantum reveals low heterogeneity among populations and its recent old world origin," PLoS Neglected Tropical Diseases, vol. 5, no. 6, Article ID el155, 2011.

[6] Brazil Ministry of Health, Department of Health, and Secretary of Surveillance in Health, Manual of Surveillance and Control of Visceral Leishmaniasis, Publishing House of the Ministry of Health, Brasilia, Brazil, 2006.

[7] Brazil Ministry of Health, (SINAN/SVS), 2014, http://dtr2004 .saude.gov.br/sinanweb/tabnet/dh?sinan/leishvi/bases/leishvbr .def.

[8] Instituto Brasileiro de Geografia e Estatística (IBGE), "Minas Gerais: IBGE,” 2010, http://cidades.ibge.gov.br/xtras/perfil.php? lang $=\& \operatorname{codmun}=313460$. 
[9] P. C. Eterovick and G. W. Fernandes, "Tadpole distribution within montane meadow streams at the Serra do Cipó, southeastern Brazil: ecological or phylogenetic constraints?" Journal of Tropical Ecology, vol. 17, no. 5, pp. 683-693, 2001.

[10] H. Pugedo, R. A. Barata, J. C. França-Silva, and E. S. Dias, "HP: an improved model of sucction light trap for the capture of small insects," Revista da Sociedade Brasileira de Medicina Tropical, vol. 38, no. 1, pp. 70-72, 2005.

[11] D. G. Young and M. A. Duncan, "Guide to the identification and geographic distribution of Lutzomyia sandflies in Mexico, the West Indies, Central and South America (Diptera: Psychodidae)," Memoirs of the American Entomological Institute, vol. 54, pp. 1-881, 1994.

[12] E. A. B. Galati, V. L. B. Nunes, E. T. Oshiro, and F. A. Rego Jr., "New species of Phlebotominae, Lutzomyia corumbaensis, sp. n. (Diptera, Psychodidae) of the Lutzomyia cortelezzii complex," Revista Brasileira de Entomologia, vol. 33, pp. 765-475, 1989.

[13] R. M. Lins, S. G. Oliveira, N. A. Souza et al., "Molecular evolution of the cacophony IVS6 region in sandflies," Insect Molecular Biology, vol. 11, no. 2, pp. 117-122, 2002.

[14] G. J. J. M. van Eys, G. J. Schoone, N. C. M. Kroon, and S. B. Ebeling, "Sequence analysis of small subunit ribosomal RNA genes and its use for detection and identification of Leishmania parasites," Molecular and Biochemical Parasitology, vol. 51, no. 1, pp. 133-142, 1992.

[15] I. Cruz, C. Cañavate, J. M. Rubio et al., "A nested polymerase chain reaction (Ln-PCR) for diagnosing and monitoring Leishmania infantum infection in coinfected patients with human immunodeficiency virus," Transactions of the Royal Society of Tropical Medicine and Hygiene, vol. 96, pp. S185-S189, 2002.

[16] I. Cruz, C. Chicharro, J. Nieto et al., "Comparison of new diagnostic tools for management of pediatric mediterranean visceral leishmaniasis," Journal of Clinical Microbiology, vol. 44, no. 7, pp. 2343-2347, 2006.

[17] B. R. Paiva, N. F. C. Secundino, J. C. Nascimento et al., "Detection and identification of Leishmania species in fieldcaptured phlebotomine sandflies based on mini-exon gene PCR," Acta Tropica, vol. 99, no. 2-3, pp. 252-259, 2006.

[18] A. C. Gomes, E. X. Rabello, J. L. Santos, and E. A. Galati, "Ecological aspects of American cutaneous leishmaniasis. I. Experimental study of the frequency of Phlebotomus in artificial ecotopes with special reference to Psychodopygus intermedius," Revista de Saude Publica, vol. 14, no. 4, pp. 540-556, 1980.

[19] G. M. de Aguiar and T. Soucasaux, "Ecological aspects of phlebotomus of the parque nacional da serra dos Orgãos, Rio de Janeiro. I. Monthly frequency in human baits (diptera, psychodidae, phlebotominae)," Memorias do Instituto Oswaldo Cruz, vol. 79, no. 2, pp. 197-209, 1984.

[20] M. F. Ximenes, M. F. Souza, and E. G. Castellón, "Density of sand flies (Diptera: Psychodidae) in domestic and wild animal shelters in an area of visceral leishmaniasis in the state of Rio Grande do Norte, Brazil," Memorias do Instituto Oswaldo Cruz, vol. 94, no. 4, pp. 427-432, 1999.

[21] O. D. Salomón, G. C. Rossi, B. Cousiño et al., "Phlebotominae sand flies in Paraguay. Abundance distribution in the Southeastern region," Memorias do Instituto Oswaldo Cruz, vol. 98, no. 2, pp. 185-190, 2003.

[22] R. A. Barata, J. C. F. D. Silva, R. T. D. Costa et al., "Phlebotomine sand flies in Porteirinha, an area of American visceral leishmaniasis transmission in the State of Minas Gerais, Brazil," Memorias do Instituto Oswaldo Cruz, vol. 99, no. 5, pp. 481-487, 2004.
[23] C. M. de Souza, J. E. Pessanha, R. A. Barata, É. M. Monteiro, D. C. Costa, and E. S. Dias, "Study on phlebotomine sand fly (Diptera: Psychodidae) fauna in Belo Horizonte, state of Minas Gerais, Brazil," Memorias do Instituto Oswaldo Cruz, vol. 99, no. 8, pp. 795-803, 2004.

[24] M. C. de Resende, M. C. V. Camargo, J. R. M. Vieira et al., "Seasonal variation of Lutzomyia longipalpis in Belo Horizonte, State of Minas Gerais," Revista da Sociedade Brasileira de Medicina Tropical, vol. 39, no. 1, pp. 51-55, 2006.

[25] E. S. Dias, J. C. França-Silva, J. C. Da Silva et al., "Sandflies (Diptera: Psychodidae) in an outbreak of cutaneous leishmaniasis in the State of Minas Gerais," Revista da Sociedade Brasileira de Medicina Tropical, vol. 40, no. 1, pp. 49-52, 2007.

[26] A. G. Oliveira, E. A. B. Galati, C. E. Fernandes, M. E. C. Dorval, and R. P. Brazil, "Seasonal variation of Lutzomyia longipalpis (Lutz \& Neiva, 1912) (Diptera: Psychodidae: Phlebotominae) in endemic area of visceral leishmaniasis, Campo Grande, state of Mato Grosso do Sul, Brazil," Acta Tropica, vol. 105, no. 1, pp. 5561, 2008.

[27] E. M. Michalsky, J. C. França-Silva, R. A. Barata et al., "Phlebotominae distribution in Janaúba, an area of transmission for visceral leishmaniasis in Brazil," Memorias do Instituto Oswaldo Cruz, vol. 104, no. 1, pp. 56-61, 2009.

[28] É. M. Michalsky, C. L. Fortes-Dias, J. C. França-Silva, M. F. Rocha, R. A. Barata, and E. S. Dias, "Association of Lutzomyia longipalpis (Diptera: Psychodidae) population density with climate variables in Montes Claros, an area of American visceral leishmaniasis transmission in the state of Minas Gerais, Brazil," Memórias do Instituto Oswaldo Cruz, vol. 104, no. 8, pp. 11911193, 2009.

[29] E. F. Rangel and R. Lainson, "Proven and putative vectors of American cutaneous leishmaniasis in Brazil: aspects of their biology and vectorial competence," Memorias do Instituto Oswaldo Cruz, vol. 104, no. 7, pp. 937-954, 2009.

[30] A. C. Gomes and E. A. Galati, "Phlebotominae from Londrina, Paraná (Brazil) and ecological observations of some species," Revista de Saude Publica, vol. 11, no. 2, pp. 284-287, 1977.

[31] W. Mayrink, P. Williams, M. V. Coelho et al., "Epidemiology of dermal leishmaniasis in the Rio Doce Valley, State of Minas Gerais, Brazil," Annals of Tropical Medicine and Parasitology, vol. 73, no. 2, pp. 123-137, 1979.

[32] V. M. Passos, A. L. Falcão, M. C. Marzochi et al., "Epidemiological aspects of American cutaneous leishmaniasis in a periurban area of the metropolitan region of Belo Horizonte, Minas Gerais, Brazil.," Memorias do Instituto Oswaldo Cruz, vol. 88, no. 1, pp. 103-110, 1993.

[33] J. D. Andrade Filho, A. P. Carneiro, M. L. Lima et al., "Sand flies in Timóteo, Minas Gerais, Brazil," Cadernos de Saúde Pública, vol. 13, pp. 767-770, 1997.

[34] D. R. D. Santos, A. R. D. Santos, E. S. D. Santos, O. D. Oliveira, L. P. Poiani, and A. M. D. Silva, "Observing diurnal habits of Nyssomyia whitmani (Diptera: Psychodidae) in the urban area of Maringá, Paraná, Brazil," Epidemiologia e Serviços de Saúde, vol. 18, pp. 227-236, 2009.

[35] G. B. M. L. Maciel and N. A. Missawa, "Sandflies (Diptera: Psychodidae) in indigenous villages in the State of Mato Grosso," Revista da Sociedade Brasileira de Medicina Tropical, vol. 42, no. 5, pp. 597-602, 2009.

[36] A. C. Azevedo, E. F. Rangel, E. M. Costa, J. David, A. W. Vasconcelos, and U. G. Lopes, "Natural infection of Lutzomyia (Nyssomyia) whitmani (Antunes \& Coutinho, 1939) by Leishmania of the braziliensis complex in Baturité, Ceará State, 
Northeast Brazil," Memorias do Instituto Oswaldo Cruz, vol. 85, no. 2, p. 251, 1990.

[37] L. Saraiva, J. D. A. Filho, S. de Oliveira Silva, A. S. R. de Andrade, and M. N. Melo, "The molecular detection of different Leishmania species within sand flies from a cutaneous and visceral leishmaniasis sympatric area in Southeastern Brazil," Memorias do Instituto Oswaldo Cruz, vol. 105, no. 8, pp. 10331039, 2010.

[38] E. A. B. Galati, V. L. B. Nunes, M. E. C. Dorval et al., "Study of the phlebotomines (Diptera, Psychodidae), in area of cutaneous leishmaniasis in the Mato Grosso do Sul State, Brazil," Revista de Saude Publica, vol. 30, no. 2, pp. 115-128, 1996.

[39] R. P. Brazil, L. V. Carneiro, J. D. Andrade Filho, J. C. M. Alves, and A. L. Falcão, "Biology of Lutzomyia lenti (Mangabeira) (Diptera: Psychodidae)," Anais da Sociedade Entomologica do Brasil, vol. 26, pp. 191-193, 1997.

[40] C. Margonari, R. P. Soares, J. D. Andrade-Filho et al., "Phlebotomine sand flies (Diptera: Psychodidae) and Leishmania infection in Gafanhoto Park, Divinópolis, Brazil," Journal of Medical Entomology, vol. 47, no. 6, pp. 1212-1219, 2010.

[41] A. C. Gomes and E. A. Galati, "Ecological aspects of the American cutaneous leishmaniasis. 7. Phlebotomus vectorial capacity in the primitive forest environment of the Serra do Mar system, Vale do Ribeira region, State of São Paulo, Brazil," Revista de Saúde Pública, vol. 23, pp. 136-142, 1989.

[42] V. L. F. de Camargo-Neves, A. de Castro Gomes, and J. L. F. Antunes, "Correlation of the presence of phlebotominae species (Diptera: Psichodidae) with records of American tegumentary leishmaniasis cases in the state of São Paulo, Brazil," Revista da Sociedade Brasileira de Medicina Tropical, vol. 35, no. 4, pp. 299306, 2002.

[43] L. Saraiva, J. Dos Santos Lopes, G. B. Machado Oliveira, F. De Assis Batista, A. L. Falcão, and J. D. Andrade Filho, "Study of the sand flies in American cutaneous leishmaniasis area, in the municipality of Alto Caparaó and Caparaó, Minas Gerais State," Revista da Sociedade Brasileira de Medicina Tropical, vol. 39, no. 1, pp. 56-63, 2006.

[44] F. S. Silva, L. P. C. de Carvalho, F. P. Cardozo, J. L. P. Moraes, and J. M. M. Rebêlo, "Sand flies (Diptera: Psychodidae) in a cerrado area of the Maranhão state, Brazil," Neotropical Entomology, vol. 39, no. 6, pp. 1032-1038, 2010.

[45] S. B. Pessoa and J. O. Coutinho, "Natural infection of Phlebotomus pessoai by leptomonas forms probably from Leishmania braziliensis," Revista de Biologia e Higiene, vol. 10, pp. 139-142, 1940.

[46] S. B. Pessoa and J. O. Coutinho, "Natural and experimental infection of phlebotomine by Leishmania braziliensis in the state of São Paulo," Hospital, vol. 20, pp. 49-63, 1941.

[47] G. M. L. Carvalho, J. D. Andrade Filho, A. L. Falcão, A. C. V. M. Rocha Lima, and C. M. F. Gontijo, "Naturally infected Lutzomyia sand flies in a Leishmania-endemic area of Brazil," Vector-Borne and Zoonotic Diseases, vol. 8, no. 3, pp. 407-414, 2008.

[48] L. Saraiva, G. M. L. Carvalho, C. M. F. Gontijo et al., "Natural infection of Lutzomyia neivai and Lutzomyia sallesi (Diptera: Psychodidae) by Leishmania infantum chagasi in Brazil," Journal of Medical Entomology, vol. 46, no. 5, pp. 1159-1163, 2009.

[49] J. Rosa, D. P. Pereira, R. P. Brazil, J. D. A. Filho, O. Salomón, and E. Szelag, "Natural infection of cortelezzii complex (Diptera: Psychodidae: Phlebotominae) with Leishmania braziliensis in Chaco, Argentina," Acta Tropica, vol. 123, no. 2, pp. 128-131, 2012.
[50] J. O. Coutinho and M. P. Barreto, "Bionomic data of "Phlebotomus fischeri” Pinto, 1926 (Diptera: Psychodidae)," Revista Brasileira de Biologia, vol. 1, pp. 423-429, 1941.

[51] M. B. de Souza, M. C. de Almeida Marzochi, R. W. de Carvalho et al., "Absence of Lutzomyia longipalpis in some endemic visceral leishmaniasis areas in Rio de Janeiro municipality," Cadernos de Saúde Pública, vol. 19, no. 6, pp. 1881-1885, 2003.

[52] M. R. de Carvalho, H. F. Valença, F. J. da Silva et al., "Natural Leishmania infantum infection in Migonemyia migonei (França, 1920) (Diptera:Psychodidae:Phlebotominae) the putative vector of visceral leishmaniasis in Pernambuco State, Brazil," Acta Tropica, vol. 116, no. 1, pp. 108-110, 2010.

[53] O. D. Salomón, M. G. Quintana, G. Bezzi, M. L. Morán, E. Betbeder, and D. V. Valdéz, "Lutzomyia migonei as putative vector of visceral leishmaniasis in La Banda, Argentina," Acta Tropica, vol. 113, no. 1, pp. 84-87, 2010.

[54] S. P. Brandão-Filho, F. G. de Carvalho, M. E. de Brito, F. A. Almeida, and L. A. Nascimento, "American cutaneous leishmaniasis in Pernambuco, Brazil: eco-epidemiological aspects in “Zona da Mata" region," Memórias do Instituto Oswaldo Cruz, vol. 89, no. 3, pp. 445-449, 1994.

[55] O. D. Salomón, S. A. Acardi, D. J. Liotta et al., "Epidemiological aspects of cutaneous leishmaniasis in the Iguazú falls area of Argentina," Acta Tropica, vol. 109, no. 1, pp. 5-11, 2009.

[56] R. Killick-Kendrick, "Phlebotomine vectors of the leishmaniases: a review," Medical and Veterinary Entomology, vol. 4, no. 1, pp. 1-24, 1990.

[57] J. C. Miranda, E. Reis, A. Schriefer et al., "Frequency of infection of Lutzomyia phlebotomines with Leishmania braziliensis in a Brazilian endemic area as assessed by pinpoint capture and polymerase chain reaction," Memorias do Instituto Oswaldo Cruz, vol. 97, no. 2, pp. 185-188, 2002.

[58] F. D. O. Lara-Silva, R. A. Barata, É. M. Michalsky et al., "Rattus norvegicus (rodentia: Muridae) infected by Leishmania (Leishmania) infantum (syn. Le. chagasi) in Brazil," BioMed Research International, vol. 2014, Article ID 592986, 7 pages, 2014. 

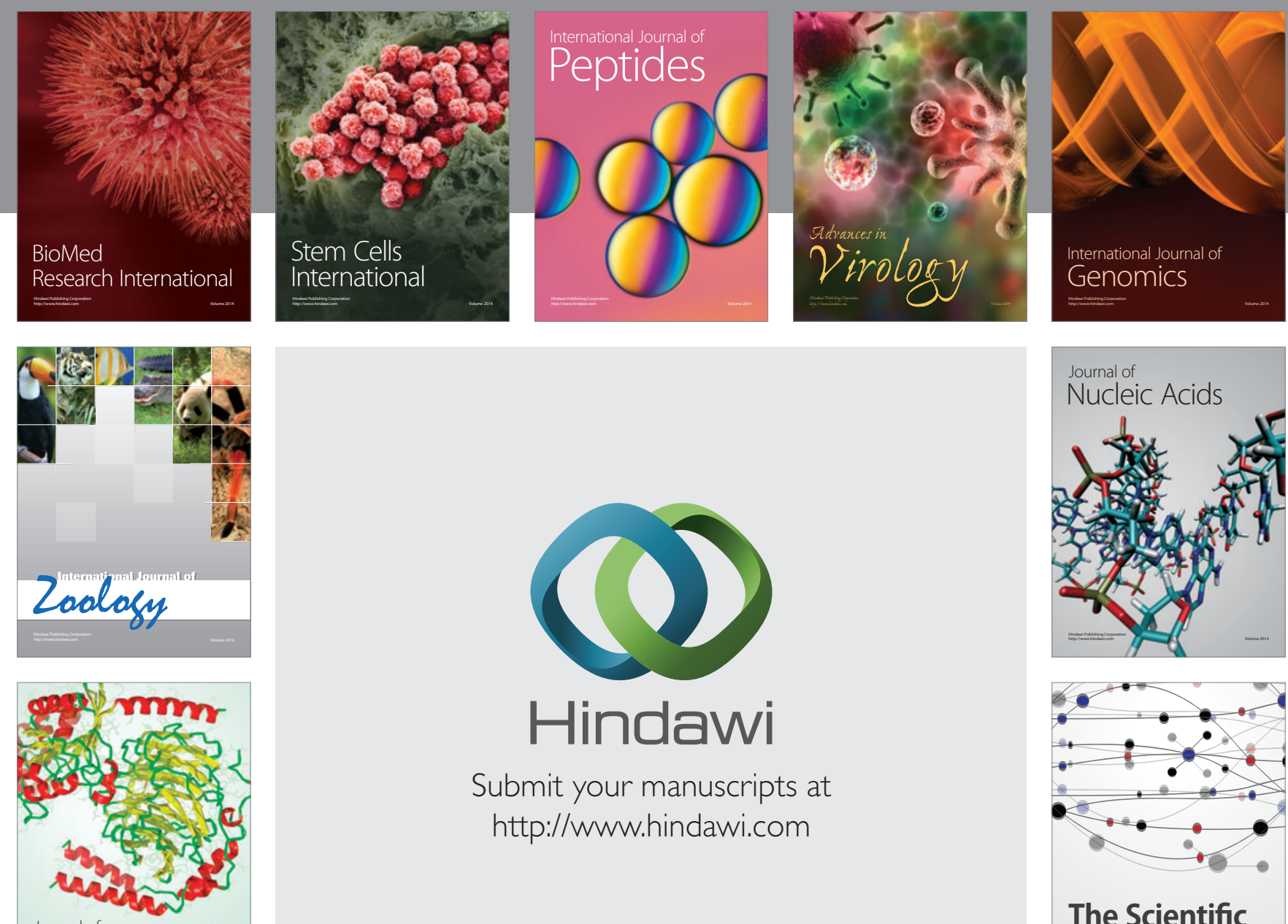

Submit your manuscripts at

http://www.hindawi.com

Journal of
Signal Transduction
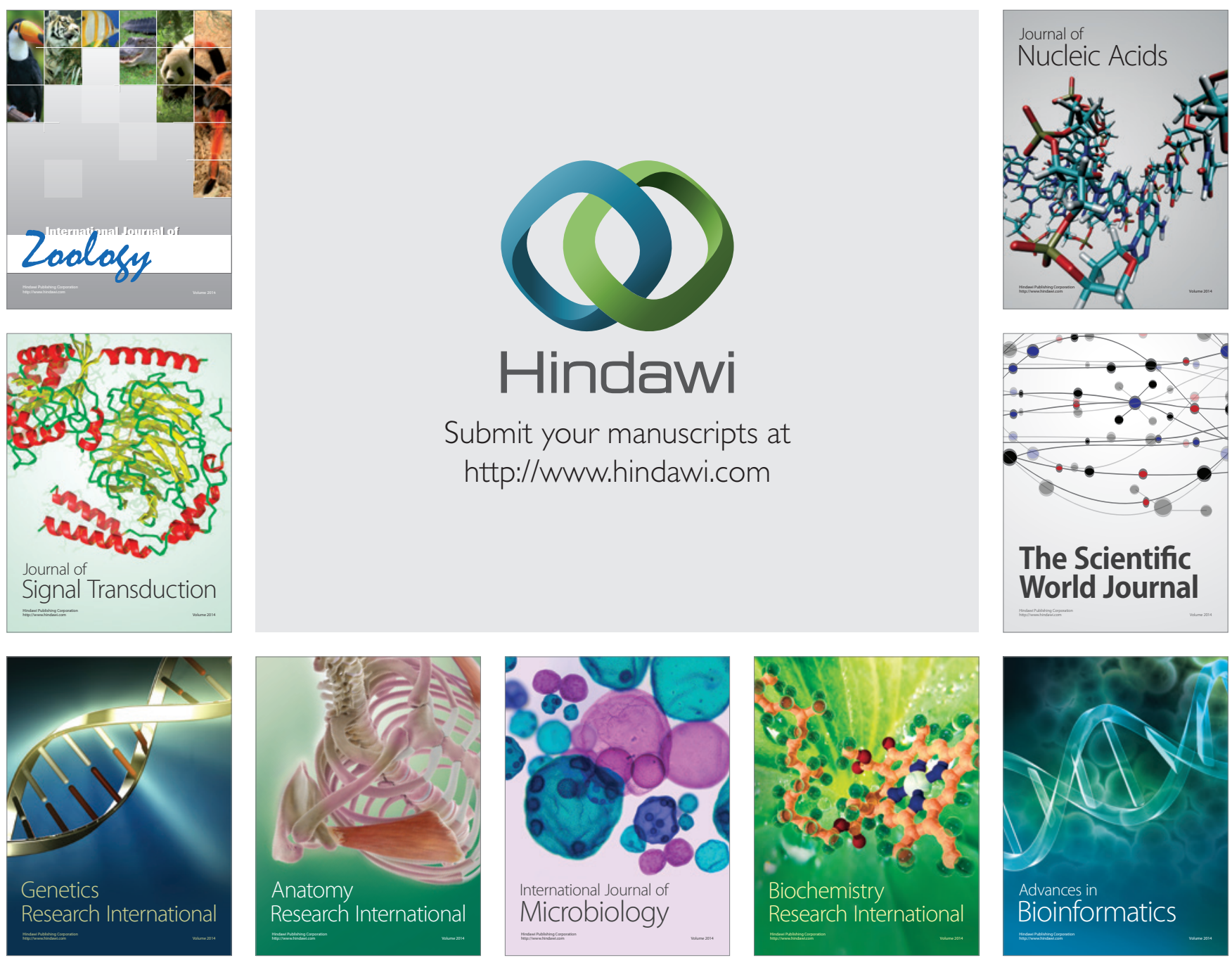

The Scientific World Journal
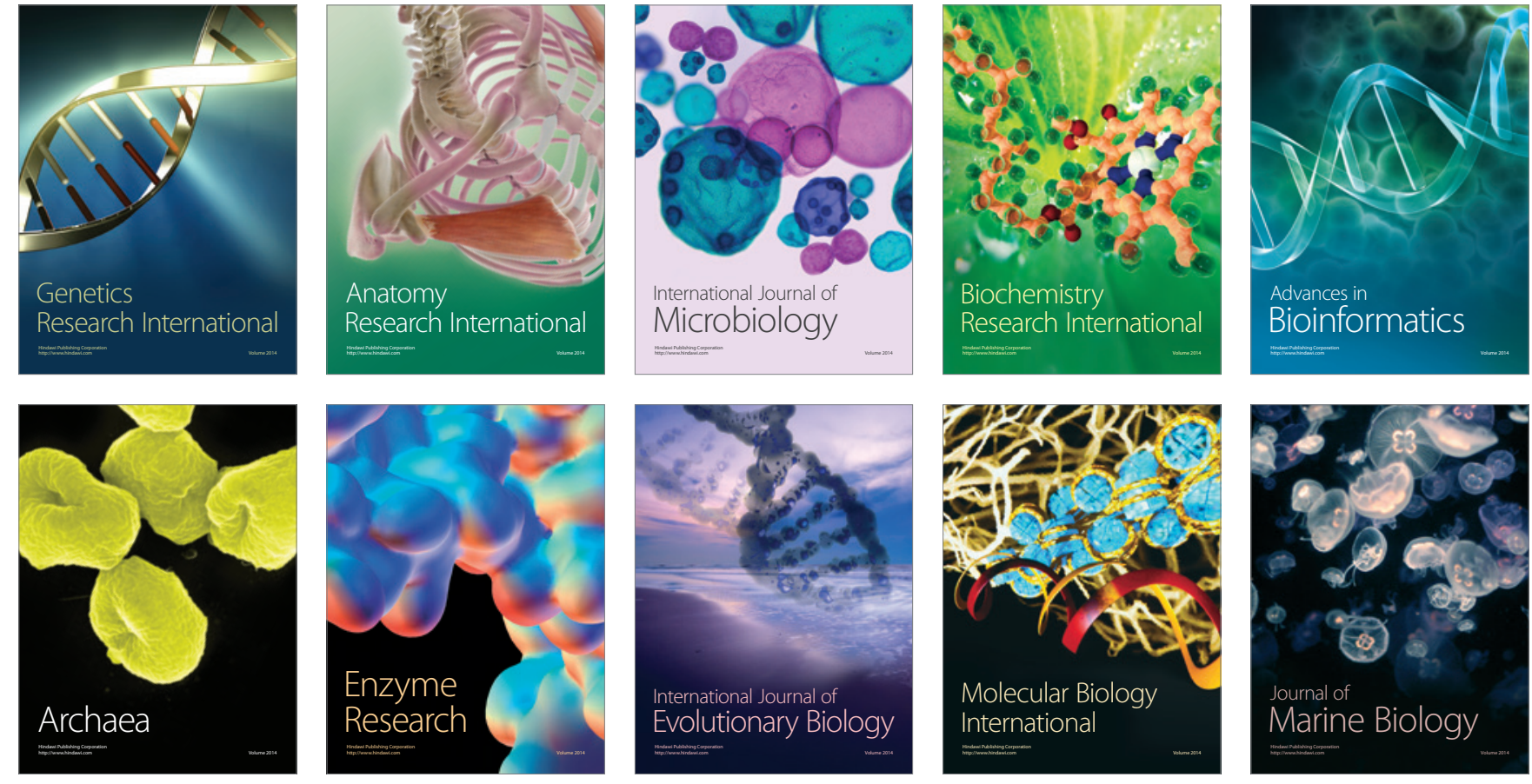